\title{
DIFFERENCES IN MUSCLE OXYGENATION BETWEEN YOUNG AND MIDDLE-AGED RECREATIONALLY ACTIVE MEN DURING HIGH-VOLUME RESISTANCE EXERCISE
}

\author{
Yftach Gepner ${ }^{1,2}$, Adam J. Wells ${ }^{1}$, Joseph A. Gordon ${ }^{1}$, Eliott Arroyo ${ }^{1}$, Alyssa N. Varanoske ${ }^{1}$, \\ Nicholas A. Coker ${ }^{1}$, David H. Fukuda ${ }^{1}$, Jeffrey R. Stout ${ }^{1}$, and Jay R. Hoffman ${ }^{1}$ \\ ${ }^{I}$ Institute of Exercise Physiology and Wellness, Sport and Exercise Science; \\ University of Central Florida, Orlando, FL, USA \\ ${ }^{2}$ School of Public Health, Sackler Faculty of Medicine and Sylvan Adams Sports Institute, \\ Tel Aviv University
}

Original scientific paper

https://doi.org/10.26582/k.51.1.4

\begin{abstract}
:
The aim of this study was to compare muscle oxygenation of the vastus lateralis during a high-volume isokinetic resistance exercise protocol (HVP) between young adult (YA) and middle-aged adult (MA) men. Twenty recreationally trained men were assigned to either the YA (age 21.8 \pm 2.0 years, body mass $90.7 \pm 11.6$ $\mathrm{kg}$, body height $179 \pm 4.7 \mathrm{~cm}$ ) or MA (age $47.0 \pm 4.4$ years, body mass $96.1 \pm 21.6 \mathrm{~kg}$, body height $177 \pm 7.7 \mathrm{~cm}$ ) group. The HVP consisted of eight sets of 10 repetitions of unilateral isokinetic concentric knee extension and eccentric knee flexion at $60^{\circ} \cdot \mathrm{s}^{-1}$. Changes in tissue hemoglobin saturation index (TSI), tissue oxygenated hemoglobin concentration $\left(\mathrm{O}_{2} \mathrm{Hb}\right)$, deoxygenated hemoglobin $(\mathrm{HHb})$, and muscle oxidation index $\left(\mathrm{O}_{2} \mathrm{Hb}-\mathrm{HHb}\right)$ were measured during the exercise session using the near-infrared spectroscopy (NIRS). Data were analyzed using two-way mix factorial analyses of variance. Prior to exercise, TSI was significantly greater $(\mathrm{p}=.024)$ for YA compared to MA. Significant decreases in $\mathrm{O}_{2} \mathrm{Hb}$ and $\mathrm{O}_{2} \mathrm{Hb}-\mathrm{HHb}$ and increases in $\mathrm{HHb}$ were observed during each of the eight sets relative to the rest periods $(\mathrm{p}<.05)$ for both groups. The average change during the eight sets of the HVP revealed a significantly higher $(p=.036)$ level of HHb and a lower $(p=.029)$ level of $\mathrm{O}_{2} \mathrm{Hb}-\mathrm{HHb}$ for MA compared to YA. A significant negative correlation was also noted at baseline between $\mathrm{O}_{2} \mathrm{Hb}-\mathrm{HHb}$ index and the cross-sectional area of the vastus lateralis muscle $(\mathrm{r}=-.45, \mathrm{p}=.045)$. During a highvolume resistance exercise, MA experienced reduced muscle oxygen saturation levels compared to YA. These results may be attributed to reductions in local tissue oxidative capacity and reduced blood delivery occurring during middle-age, and possibly due to group differences in muscle morphology.
\end{abstract}

Key words: oxygen kinetics, aging, resistance exercise, near-infrared spectroscopy

\section{Introduction}

Decreased aerobic power is one of the physiological changes associated with aging, and is related to reductions in mitochondrial density and oxidative enzyme activity (Conley, Jubrias, \& Esselman, 2000). These changes may also be associated with alterations in muscle oxygenation, which may limit $\mathrm{O}_{2}$ consumption in the muscle during exercise in older adults (Kirkendall \& Garrett, 1998; Kutsuzawa, Shioya, Kurita, Haida, \& Yamabayashi, 2001). Several studies have compared the muscle oxygenation response during exercise and recovery between young and older adults (DeLorey, Paterson, \& Kowalchuk, 2007; Ichimura, et al., 2006; Kutsuzawa, et al., 2001; Lin, Lin, Ho, $\&$ Chen, 2014). A greater muscle deoxygenation during muscle contraction has been reported among older adults compared to young adults (DeLorey, et al., 2007; Ichimura et al., 2006). In addition, others have demonstrated a prolonged recovery time for muscle reoxygenation following submaximal exercise with increased age (Kutsuzawa, et al., 2001; Lin, et al., 2014). Although age-associated alterations in muscle oxygenation kinetics and oxygen consumption have been observed during exercise, no investigations to our knowledge have specifically compared younger to middle-aged adults. It is currently unknown whether the differences seen during aging begin during middle-age (40-60 years). Furthermore, most investigations comparing differences in muscle oxygenation between older and younger adults have primarily examined 
submaximal, endurance exercise. Whether these differences are also apparent during resistance exercise is not well understood.

Near-infrared spectroscopy (NIRS) is a noninvasive technique that provides information regarding changes in tissue oxygenation based on oxygen-dependent absorption characteristics of infrared light in the range of 600-900nm (Grassi \& Quaresima, 2016; Jones, Hesford, \& Cooper, 2013; Vercruyssen, et al., 2014). The benefit of NIRS is in its ability to measure the instantaneous oxygenation response in a less restricted environment, which is suitable for evaluating exercise tasks involving both static and dynamic exercises (Hoffman, et al., 2007; Pereira, Gomes, \& Bhambhani, 2007). Previous research has demonstrated no differences in muscle deoxygenation between high-intensity versus high-volume resistance training programs (Hoffman, et al., 2003). However, a greater duration of a high-volume resistance training (15 repetitions at $60 \%$ of the participants' maximal strength) has been shown to result in a significant delay in muscle reoxygenation. Only one study appears to have compared older ( $73 \pm 7$ years) to younger ( $22 \pm 2$ years) adults on a resistance type exercise protocol (Hart, et al., 2015). Participants were required to perform a constant load at approximately $40 \%$ of maximum plantar flexion work rate for five minutes. The investigators reported no age-related differences in muscle deoxygenation or reoxygenation. Whether this occurs at higher exercise intensities, is not known.

The purpose of this study was to compare muscle oxygenation of the vastus lateralis during a high-volume isokinetic resistance exercise protocol between young to middle-aged adult men. We hypothesized that the magnitude of oxygenation and deoxygenation during the exercise protocol would be different between the middle-aged and young men.

\section{Methods}

\section{Participants}

Twenty recreationally active adult men volunteered to participate in this study. Participants were divided into either the young adult group (YA, $\mathrm{n}=10$, age $21.8 \pm 2.04$ years, body mass $90.7 \pm 11.6 \mathrm{~kg}$, body height $179 \pm 4.7 \mathrm{~cm}$ ) or the middle age group (MA, $\mathrm{n}=10$, age $47.0 \pm 4.45$ years, body mass $96.0 \pm 21.6$ $\mathrm{kg}$, body height $177 \pm 7.7 \mathrm{~cm}$ ). Inclusion criteria required participants to be men between the ages of 18-30 years (YA group) or between 40-60 years (MA group), and be recreationally active (e.g., 150 minutes of exercise per week including resistance exercise) for at least six months as defined by the American College of Sports Medicine (Haskell, et al., 2007). All participants were free of any physical limitations that might have affected perfor- mance. Additionally, all participants were free of any medications, performance-enhancing drugs and any dietary supplements with ergogenic effects (e.g., creatine, beta-alanine, etc.) as determined by a health and activity questionnaire. The study was conducted with the approval by the New England Independent Review Board before participant enrollment (NEIRB\# 120160966), and all subjects gave written informed consent before testing.

\section{Testing procedure}

Both groups reported to the Human Performance Laboratory (HPL) on two separate occasions. On the first visit, participants reported to the HPL following a 2-hour fast. Anthropometric assessments were performed including the body height, body weight, and body composition determination. Following anthropometric assessments, participants performed a standardized warm-up consisting of 5 minutes of self-paced pedaling on a cycle ergometer at $50 \mathrm{~W}$. Following the warm-up, participants completed a familiarization protocol on the isokinetic device (S4, Biodex Medical System, Inc., New York, NY, USA). On the second visit, participants arrived following a 10-hour fast. There were at least 48 hours between the first and the second visits, with no more than seven days between them. Following the previously described warm-up, participants performed a high-volume isokinetic resistance exercise protocol (HVP). Ultrasound assessments were obtained before and immediately following the exercise protocol, and near-infrared spectroscopy (NIRS) measures were performed throughout the duration of the HVP.

\section{Anthropometric measurements}

Body height $( \pm 0.1 \mathrm{~cm})$ and body weight $( \pm 0.1 \mathrm{~kg})$ were measured by a standard wall-mounted stadiometer (Patient Weighing Scale, Model 500 KL, Pelstar, Alsip, IL, USA). Body composition was measured using a direct segmental multi-frequency bioelectrical impedance analyzer (BIA) via InBody (Model 770, InBody Co., Ltd, Cerritos, CA, USA) according to the manufacturer's guidelines. Participants were asked to refrain from food consumption within two hours of the test and to be normally hydrated as well as to remove all jewelry and footwear, including socks, and to void the bladder before performing the test. This assessment required the participants to wipe their hands and feet with an InBody tissue to enhance electrical conductivity and reduce surface bacteria before being assessed. The participants were then instructed to stand on the InBody platform electrodes while holding two hand electrodes out to the side. They held this position for one minute as the device conducted the electrical currents through the body to determine body composition. Values for total body fat percentage were recorded. 


\section{High-volume isokinetic resistance exercise protocol (HVP)}

During the HVP participants were seated in the isokinetic dynamometer (S4, Biodex Medical System, Inc., New York, NY, USA), positioned with a hip angle of $110^{\circ}$ and strapped into the chair at the waist, shoulders, and across the thigh. The chair and dynamometer settings were adjusted for each participant to correctly align the axis of rotation with the lateral condyle of the femur. All participants were tested on their right leg, which was secured to the dynamometer arm just above the medial and lateral malleoli. The lever arm of the dynamometer was programmed to extend the participant's leg to $155^{\circ}$ of knee flexion (where $180^{\circ}$ is full extension) and flex the participant's leg to $85^{\circ}$ of flexion. Isokinetic dynamometer settings for each individual were recorded and remained consistent throughout the study. The HVP consisted of eight sets of 10 repetitions of concentric knee extension and eccentric knee flexion at $60^{\circ} \cdot \mathrm{s}^{-1}$ with three minutes of rest between sets. Participants were encouraged to provide maximal effort throughout the HVP. Work performed in each set of the HVP was calculated as the product of the mean power of each kick over the time needed to complete the kick. Total work done was calculated as the sum of the work performed in each of the eight sets of 10 repetitions during the HVP. The highest peak torque (PKT) and average torque (AVGT) were recorded

\section{Near-infrared spectroscopy measurement (NIRS)}

A portable NIRS apparatus (PortaMon, Artinis Medical Systems, Gelderland, the Netherlands) was used in this study. The NIRS was placed over the vastus lateralis muscle of the right leg at a location equidistant from the greater trochanter of the femur and the muscle's distal connection to the quadriceps tendon according the manufacturer's guidelines. The location of the probe was recorded by noting distances from the anatomic landmark. The device was held securely in place with sports tape covering to prevent contamination from ambient light. The NIRS device was connected to a personal computer via Bluetooth ${ }^{\mathrm{TM}}$ technology for data acquisition. This non-invasive method allows an estimation of changes in oxygenation in tissues by assessing changes in the concentration of oxygenated and deoxygenated hemoglobin and myoglobin (Mancini, et al., 1994). Changes in tissue oxygenated hemoglobin concentration $(\mathrm{O} 2 \mathrm{Hb})$, deoxygenated hemoglobin concentration ( $\mathrm{HHb}$ ), and tissue hemoglobin saturation index (TSI) were measured using the difference in absorption characteristics of light at 750 and $850 \mathrm{~nm}$. Muscle oxidation index was calculated by taking the difference between each $\mathrm{O} 2 \mathrm{Hb}$ and $\mathrm{HHb}$ values $(\mathrm{O} 2 \mathrm{Hb}-\mathrm{HHb})$ that occurred during testing (Hamaoka, McCully, Quaresima,
Yamamoto, \& Chance, 2007). Values for $\mathrm{O} 2 \mathrm{Hb}$, $\mathrm{HHb}$, and $\mathrm{O} 2 \mathrm{Hb}-\mathrm{HHb}$ were reported as a change from baseline (three minutes averaging before test) in micromolar units $(\mu \mathrm{M})$. Other study has found ICC's $>0.9$ for those measurements using the same device (Adami, et al., 2017). The influence of highvolume resistance training on the muscle oxidation kinetics was investigated during the HVP.

\section{Ultrasound assessment}

To provide a perspective of potential differences in muscle morphology on changes in muscle oxygenation, muscle ultrasound assessments were performed on the vastus lateralis of the right leg before and following the HVP. Assessments were performed as previously described (Wells, et al., 2014). Participants were asked to lay supine on an examination table with both legs fully extended for a minimum of five minutes to allow fluid shifts to occur (Arroyo, et al., 2016). Before image collection, all anatomical locations of interest were identified using standardized landmarks for the vastus lateralis. After determining the desired anatomical position, a $12 \mathrm{MHz}$ linear probe (General Electric LOGIQ P5, Wauwatosa, WI, USA), coated with the transmission gel (Aquasonic ${ }^{\circledR}$ 100, Parker Laboratories, Inc., Fairfield, NJ), was positioned on the surface of the skin to collect the ultrasound image. For all ultrasound measurements, depth was set at $6.0 \mathrm{~cm}$, gain at $50 \mathrm{~dB}$, and the dynamic range was set at 72 . All ultrasound images were analyzed offline using image analysis software (ImageJ, version $1.45 \mathrm{~s}$ ) available from the National Institutes of Health (NIH, Bethesda, MD, USA). Muscle thickness measures were obtained using a longitudinal B-mode image from the average of two consecutive images. For each image, a single perpendicular line was drawn from the superficial aponeurosis to the deep aponeurosis. The cross-sectional area measures were obtained using a transverse sweep in the extended field of view mode from two consecutive images. For each image, muscle area was assessed using the polygon tracking tool to include as much lean tissue as possible while excluding bone and surrounding fascia. The average of the two measures was used for statistical analyses. Echo intensity was measured from the transverse panoramic images using the standard histogram function, which measures the greyscale of each pixel in arbitrary units (AU). Echo intensity is suggested to provide a measure of muscle 'quality' by delineating the ratio of contractile to non-contractile tissues within the ultrasound image (Tsukagoshi, Tateuchi, Fukumoto, Okumura, \& Ichihashi, 2012). Test-related reliability for all ultrasound measurements was determined by using ten subjects and measured 24-48 hours apart. Using the recommendation of Weir (Weir, 2005), the intraclass correlation coefficient (ICC) for muscle thickness was 
$0.996(\mathrm{SEM}=0.851 \mathrm{~cm})$, ICC for cross-sectional area was $0.973\left(\mathrm{SEM}=0.092 \mathrm{~cm}^{2}\right)$ and ICC for echo intensity was 0.996 (SEM=1.455), which was similar to values reported previously (Wells, et al., 2014). All ultrasound measurements and image analyses were performed by the same investigator and were blinded for the study group and time points.

\section{Dietary assessment}

Participants were asked to provide a 3-day dietary recall and to maintain their regular diet. FoodWorks nutrient analysis software (McGrawHill, New York, NY, USA) was used to analyze the self-reported dietary recalls for the total calorie intake and macronutrient portions (carbohydrate, protein, and fat). Additionally, participants were also asked to abstain from any nonsteroidal antiinflammatory drugs, cryotherapy, or any other agents that may affect inflammation or recovery.

\section{Statistical analysis}

Independent-samples $t$-tests were used to identify the between-group differences at baseline and for the average changes of muscle saturation variables of the HVP. Two-way (Group x Sets) mixed factorial analysis of variance was used to assess changes of $\mathrm{O}_{2} \mathrm{Hb}, \mathrm{HHb}$, and $\mathrm{O}_{2} \mathrm{Hb}-\mathrm{HHb}$ during testing. In the event of a significant $\mathrm{F}$ ratio, Bonferroni post-hoc pairwise comparisons were used to examine the differences between the groups. TSI was analyzed via repeated measures analysis of covariance to control for baseline differences between the groups. Pearson product moment correlations were used to examine the bivariate association between the ultrasound (cross-sectional area, echo intensity, and muscle thickness) and muscle saturation variables. A criterion level of $\alpha \leq .05$ was used to determine statistical significance. Statistical analyses were performed using the statistical analysis software program SPSS (version 23, SPSS Inc., Chicago, IL, USA). All data are reported as mean \pm standard deviation.

\section{Results}

Anthropometric characteristics and daily nutrient intakes of the study groups are shown in Table 1. No significant differences in anthropometric characteristics were found between the groups $(\mathrm{p}>.05)$, except for age $(\mathrm{p}=.006)$. In addition, no significant differences at baseline were noted between the groups for peak power $(p=.79)$ and mean power $(\mathrm{p}=.77)$. Both groups consumed similar proportion of macronutrients (fat, carbohydrates, and protein) and energy intake. Peak torque comparisons between these groups have been previously reported (Gordon, et al., 2017). During isometric comparisons, YA was reported to have significantly greater average torque, rate
Table 1. Anthropometric characteristics and average daily nutrient intake

\begin{tabular}{lcc}
\hline & $\begin{array}{c}\text { Young age } \\
(\mathrm{n}=10)\end{array}$ & $\begin{array}{c}\text { Middle age } \\
(\mathrm{n}=10)\end{array}$ \\
\hline Anthropometric & $21.8 \pm 2.0$ & $47.0 \pm 4.4^{*}$ \\
Age (year) & $179.7 \pm 4.7$ & $176.9 \pm 7.7$ \\
Body height $(\mathrm{cm})$ & $90.7 \pm 11.6$ & $96.1 \pm 21.6$ \\
Body weight $(\mathrm{kg})$ & $21.5 \pm 4.1$ & $25.4 \pm 5.2$ \\
Body fat $(\%)$ & $19.8 \pm 6.1$ & $25.3 \pm 11.2$ \\
Fat mass $(\mathrm{kg})$ & $40.7 \pm 3.8$ & $40.1 \pm 6.4$ \\
Lean body mass $(\mathrm{kg})$ & $33.8 \pm 5.4$ & $32.1 \pm 6.5$ \\
Vastus lateralis CSA $\left(\mathrm{cm}^{2}\right)$ & & \\
Performance & $219 \pm 49$ & $213 \pm 56$ \\
Peak power $(\mathrm{w})$ & $139 \pm 42$ & $134 \pm 36$ \\
Mean power $(\mathrm{w})$ & & \\
Nutrient intake & $2,076 \pm 454$ & $1,975 \pm 520$ \\
Calorie intake $\left(\mathrm{kcal} \cdot \mathrm{day}{ }^{-1}\right)$ & $220.3 \pm 70.2$ & $224.8 \pm 69.1$ \\
Carbohydrate $(\mathrm{g})$ & $110.2 \pm 43.8$ & $94.8 \pm 21.6$ \\
Protein $(\mathrm{g})$ & $1.3 \pm 0.6$ & $1.0 \pm 0.3$ \\
Protein $\left(\mathrm{g} \cdot \mathrm{kg}{ }^{-1}\right)$ & $76.0 \pm 27.4$ & $78.6 \pm 32.9$ \\
\hline Fat (g) & &
\end{tabular}

Note. CSA $=$ cross-sectional area. All data are reported as mean $\pm S D .{ }^{*}=$ significantly different between the groups.

of torque development and a trend towards greater peak torque than MA. In addition, trends towards a difference were noted between the groups in peak torque during dynamic isokinetic contractions at $60^{\circ} \cdot \mathrm{s}^{-1}$.

\section{Near-infrared spectroscopy (NIRS) measurements}

Changes in TSI, $\mathrm{O}_{2} \mathrm{Hb}, \mathrm{HHb}$, and $\mathrm{O}_{2} \mathrm{Hb}-\mathrm{HHb}$ during each of the eight sets of the resistance training program are presented in Figures 1a-d. During baseline, TSI was significantly greater $(\mathrm{p}=.024)$ for YA compared to MA $(73.2 \pm 3.4 \%$ vs. $68.6 \pm 4.8 \%$, respectively). No other significant between-group differences were noted for resting $\mathrm{O}_{2} \mathrm{Hb}, \mathrm{HHb}$ and $\mathrm{O}_{2} \mathrm{Hb}-\mathrm{HHb}$ (p's $>12$ ).

After controlling for baseline differences of TSI, no significant group $\mathrm{x}$ set interaction $(\mathrm{F}=1.81$, $\mathrm{p}=0.297$ ) was found for changes in TSI. Yet, a significant main effect for sets was observed $(\mathrm{F}=44.1$, $\mathrm{p}=0.001)$. When collapsed across the groups, TSI was significantly decreased following the HVP. A comparison between the groups of the average change from baseline in TSI revealed a significant difference between the groups $(\mathrm{p}=.001)$. The average change in TSI was significantly lower in MA than YA $(-22.2 \pm 4.6 \%$ vs. $-17.7 \pm 9.4 \%$, respectively).

No significant group $\mathrm{x}$ set interaction $(\mathrm{F}=1.97$, $\mathrm{p}=.143)$ or main effect for group $(\mathrm{F}=0.89, \mathrm{p}=.356)$ was observed for changes in $\mathrm{O}_{2} \mathrm{Hb}$ during the 


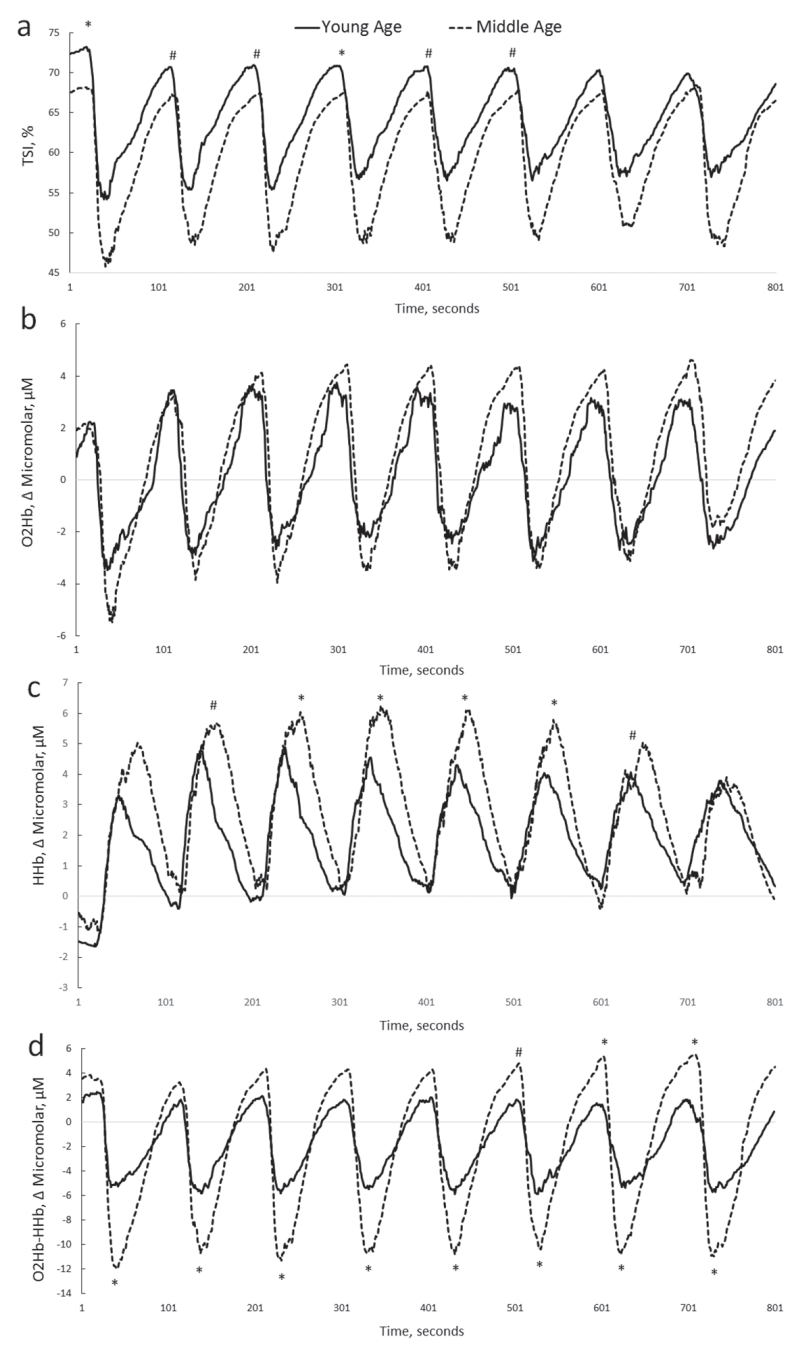

Figure 1. Muscle oxidation during eight sets of the resistance testing across the age groups.

Changes in muscle oxidation through eight sets of the resistance testing, using NIRS. Record-frequency of half a second during the measurement. Black line: young age (YA); broken line: middle age group (MA). a: tissue hemoglobin saturation index (TSI); b: oxygenated hemoglobin concentration $\left(\mathrm{O}_{2} \mathrm{Hb}\right)$; c: deoxygenated hemoglobin concentration $(\mathrm{HHb})$; $\mathrm{d}$ : hemoglobin saturation ratio $(\mathrm{O} 2 \mathrm{Hb}-\mathrm{HHb}) .{ }^{*} \mathrm{p}<.05$ for average differences between the groups for the maximal and minimal values, $\# \mathrm{p}<.01$ for average differences between the groups at baseline.

HVP. However, when collapsed across the groups, a significant main effect for sets was found for $\mathrm{O}_{2} \mathrm{Hb}$ $(\mathrm{F}=25.38, \mathrm{p}<0.001)$. Significant decreases in $\mathrm{O}_{2} \mathrm{Hb}$ were observed in each of the eight sets relative to the rest periods.

A significant group $\mathrm{x}$ set interaction was observed for $\mathrm{HHb}(\mathrm{F}=3.38, \mathrm{p}=0.031)$. $\mathrm{HHb}$ was significantly higher in MA from the third set to the sixth set compared to YA (p's<.05). A significant main effect for time was also found for $\mathrm{HHb}$ $(\mathrm{F}=4.16, \mathrm{p}=.015)$. When collapsed across the groups, a significant increase in $\mathrm{HHb}$ was observed in each of the eight sets relative to the rest periods.

No significant group $\mathrm{x}$ set interaction $(\mathrm{F}=2.41$, $\mathrm{p}=.086$ ) was observed for $\mathrm{O}_{2} \mathrm{Hb}-\mathrm{HHb}$ during the

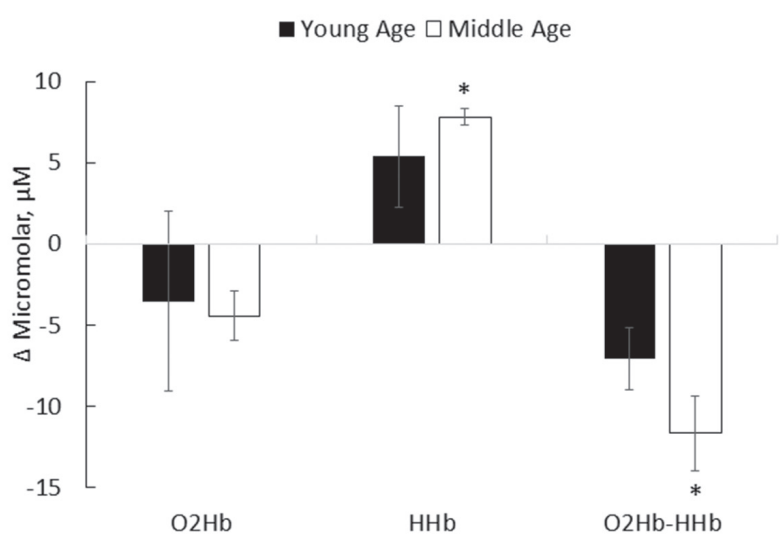

Figure 2. Mean changes of tissue oxyhemoglobin $(\mathrm{O} 2 \mathrm{Hb})$, deoxyhemoglobin (HHb), and hemoglobin saturation ratio $(\mathrm{O} 2 \mathrm{Hb}-\mathrm{HHb})$ during a high-volume resistance exercise protocol.

Mean changes of the muscle oxidation for an average of the eight sets of resistance testing. $* \mathrm{p}<.05$ for significant interaction (time $\mathrm{x}$ group). Results are reported as mean $\pm \mathrm{SD}$. $\mathrm{O}_{2} \mathrm{Hb}$ : oxygenated hemoglobin concentration; $\mathrm{HHb}$ : deoxygenated hemoglobin concentration; $\mathrm{O} 2 \mathrm{Hb}-\mathrm{HHb}$ : hemoglobin saturation ratio.

eight sets. However, a significant main effect for group was observed for $\mathrm{O}_{2} \mathrm{Hb}-\mathrm{HHb}(\mathrm{F}=6.42$, $\mathrm{p}=.021)$. When collapsed across sets, MA experienced a significantly greater $(\mathrm{p}<.05)$ decrease in $\mathrm{O}_{2} \mathrm{Hb}-\mathrm{HHb}$ during the exercise session compared to YA.

The average change in muscle saturation measures from the eight sets of the HVP can be observed in Figure 2. No significant difference $(p=.445)$ was observed for changes in $\mathrm{O}_{2} \mathrm{Hb}$. However, a significant difference ( $\mathrm{p}=.036)$ was observed for changes in the average $\mathrm{HHb}$ during the HVP. MA had a higher level of $\mathrm{HHb}$ compared to $\mathrm{YA}(7.8 \pm 0.5 \mu \mathrm{M}$ vs. $5.3 \pm 3.1 \mu \mathrm{M}$, respectively). A significant difference $(p=.029)$ between MA and YA was also observed for the average change in $\mathrm{O}_{2} \mathrm{Hb}-\mathrm{HHb}$ during the HVP (-11.3 $\pm 1.4 \mu \mathrm{M}$ vs. $-7.1 \pm 5.5 \mu \mathrm{M}$, respectively).

\section{Muscle morphology}

A significant negative correlation was noted at baseline between $\mathrm{O}_{2} \mathrm{Hb}-\mathrm{HHb}$ index and the crosssectional area of the vastus lateralis muscle $(\mathrm{r}=-.45$, $\mathrm{p}=.045$ ), but $\mathrm{O}_{2} \mathrm{Hb}-\mathrm{HHb}$ index was positively correlated with echo intensity $(r=.45, p=.044)$. In addition, muscle thickness was significantly associated with TSI $(\mathrm{r}=.48, \mathrm{p}=.031)$. The change from pre- to postexercise in the cross-sectional area of the vastus lateralis was associated with an increase in the $\mathrm{HHb}$ between sets $(\mathrm{r}=.47, \mathrm{p}=.036)$, and a decrease in $\mathrm{O} 2 \mathrm{Hb}-\mathrm{HHb}$ between sets $(\mathrm{r}=-.50, \mathrm{p}=.024)$. Echo intensity, however, was associated with an increase in $\mathrm{O}_{2} \mathrm{Hb}-\mathrm{HHb}$ between sets $(\mathrm{r}=.48, \mathrm{p}=.029)$.

\section{Discussion and conclusions}

The findings of this investigation indicated that during a high-volume isokinetic exercise, patterns 
of muscle oxygenation were different between YA and MA. Although muscle oxygenation was significantly decreased during the contraction phase of the exercise protocol, this decrease was significantly greater for MA than YA. Also, greater increases were observed for tissue deoxygenation and the muscle oxidation index in MA compared to YA. The results of this study are consistent with previous investigations reporting differences in muscle oxygen saturation when comparing YA to older adults (age $>60$ years) (Costes, et al., 1999; Ferri, et al., 2007; Miljkovic, Lim, Miljkovic, \& Frontera, 2015), suggesting that impeded delivery of $\mathrm{O}_{2}$ during a high-volume exercise may begin during middle-age.

The results of this investigation also indicated that tissue deoxygenation was increased to a greater magnitude in MA compared to YA. One of the determinants of oxygen supply to the muscle is blood flow (Gonzalez-Alonso \& Calbet, 2003), which was previously found to decrease in older adults during both exercise and recovery (Heinonen, Koga, Kalliokoski, Musch, \& Poole, 2015; Kutsuzawa, et al., 2001). Other studies have also demonstrated a reduction in leg blood flow and femoral venous $\mathrm{O}_{2}$ saturation at the same absolute workload in trained older adults compared to younger adults (DeLorey, et al., 2007; Gonzalez-Alonso \& Calbet, 2003; van Beest, et al., 2012). Although speculative, a reduced blood flow in MA participants during the exercise protocol may partially explain the differences in muscle oxygenation noted at rest between the groups. Another factor that may have influenced these differences is capillary density, which have been reported reduced during aging (Groen, et al., 2014). Decreases in the capillary density of the vastus lateralis have been observed in MA participants in one study (Mitchell, et al., 2012), but not other (Proctor, Sinning, Walro, Sieck, \& Lemon, 1995). However, capillary density was not determined in the present investigation, and no significant differences were found between the groups in cross-sectional area or muscle thickness of the vastus lateralis following the HVP. Therefore, the influence of capillary density on NIRS is still not well-understood.

Middle-aged adult men experienced a higher level of muscle deoxygenation and a lower muscle oxidation index for the average of the eight sets of exercise than the younger adults. A recent investigation comparing muscle oxygenation between young (24.8 \pm 3.6 years) and older adults $(66.5 \pm 3.0$ years) during a $20 \%$ maximal voluntary isokinetic contraction reported no significant differences between the groups at this level of effort (Lin, et al., 2014). It was suggested that the time of deoxygenation was related to muscle perfusion. However, an additional investigation examining muscle oxygenation during intense cycling exercise demonstrated that older adults (68.0 07.0 years) exhibited a longer time of deoxygenated hemoglobin kinetics (i.e., time delay + time constant) compared to young (23.0 \pm 5.0 years) adults (Murias, Kowalchuk, \& Paterson, 2010). Thus, it is possible that during a higher intensity exercise, differences in blood perfusion between muscle contractions may become a factor in $\mathrm{O}_{2}$ delivery and utilization. Results of our study suggest that this change may start during middle-age.

During the recovery phase between each repetition, MA experienced a greater muscle oxidation index, and the differences compared to the YA group gradually increased during the exercise protocol. Aging is associated with an increase in total peripheral resistance and reduced maximal heart rate, capillary density, endothelial dysfunction, and altered capillary hemodynamics, which may relate to an impeded $\mathrm{O}_{2}$ delivery during exercise (DeLorey, et al., 2007). The results of this study indicate that these changes occur independent of being recreationally active and begin during middleage. Other studies have suggested that older adults ( $>65$ years) have a longer recovery time for muscle reoxygenation due to an impaired muscle oxidative capacity (Ichimura, et al., 2006; Lin, et al., 2014). One investigation reported that older adults (age $>$ 65 years) had a $50 \%$ lower oxidative capacity per volume of quadriceps muscle than younger adults (age $<40$ years), and that this was attributable to both a decrease in mitochondrial volume and a lower oxidative capacity of the remaining mitochondria (Conley, et al., 2000). Our study, comparing muscle oxidation between YA and MA, is consistent with these previous investigations comparing younger to older adults, demonstrating a $60 \%$ difference in muscle oxidation index values between MA and YA during exercise. Moreover, significant differences between the groups were found for all changes in muscle oxidation index recorded during all eight sets. Although speculative, our results indicate that impaired $\mathrm{O}_{2}$ kinetics in MA may be the result of local tissue oxidative capacity and reduced blood delivery that begins during middle-age.

Although not a central focus of the present article, this study also examined the correlation between muscle morphology and muscle oxidation. Increased CSA following the HVP was correlated with higher levels of muscle deoxygenation and lower levels of muscle oxidation index. Taken together with the significant correlation found at baseline, these findings may suggest that increases in muscle damage during HVP may by associated with decreased oxidation in the muscle observed during incremental exercise (Koga, Rossiter, Heinonen, Musch, \& Poole, 2014; Spencer, Amano, Kondo, Kowalchuk, \& Koga, 2014). Another factor 
that may have affected muscle oxidation differences between these age groups were differences in muscle strength and power (Gordon, et al., 2017). Although not measured, it is also possible that reduction in type 2 fiber size and changes in intrinsic contractile properties of muscle fibers with aging may have also contributed to the observed results (Callahan, et al., 2014).

In short:

- Tissue hemoglobin saturation index was greater in young vs middle-age men at rest

- Muscle oxygenation response to exercise is different between young and middle-age

- Age-related changes in muscle oxygenation occurs during middle-age (40-60y)

- A reduced muscle oxygen response to exercise is seen in middle-age men

The study has several limitations that need to be acknowledged. The exercise protocol was performed on an isokinetic dynamometer, which may not have as much practical application as most resistance training programs utilize dynamic constant resistance exercises. In addition, unilateral, single-joint exercise recruited a relatively smaller muscle mass than is commonly used in most training programs. Women, who also suffer age-related changes in muscle metabolism, were not included in the study. Future research should examine modes of training specific to the typical training program of the participants.

In conclusion, the results of this investigation demonstrate that the recreationally trained middleaged adults experience a reduced muscle oxygen saturation during a high-volume resistance exercise protocol compared to the younger recreationally trained adults. These differences appear to increase in magnitude throughout the exercise and recovery phases. To the best of our knowledge, this study seems to be the first investigation to compare muscle oxygenation responses between the younger and middle-aged recreationally trained men, and it provides evidence that the age-related changes in muscle oxygenation appear to occur in middle-aged adults regardless of recreational exercise training.

\section{References}

Adami, A., Cao, R., Porszasz, J., Casaburi, R., \& Rossiter, H.B. (2017). Reproducibility of NIRS assessment of muscle oxidative capacity in smokers with and without COPD. Respiratory Physiology and Neurobiology, 235, 18-26.

Arroyo, E., Stout, J.R., Beyer, K.S., Church, D.D., Varanoske, A.N., Fukuda, D.H., \& Hoffman, J.R. (2016). Effects of supine rest duration on ultrasound measures of the vastus lateralis. Clinical Physiology and Functional Imaging, 38(1), 155-157.

Callahan, D.M., Bedrin, N.G., Subramanian, M., Berking J., Ades P.A., Toth M.J., \& Miller M.S. (2014). Age-related structural alterations in human skeletal muscle fibers and mitochondria are sex specific: Relationship to singlefiber function. Journal of Applied Physiology, 116(12), 1582-1592.

Conley, K.E., Jubrias, S.A., \& Esselman, P.C. (2000). Oxidative capacity and ageing in human muscle. Journal of Physiology, 526(Pt 1), 203-210.

Costes, F., Denis, C., Roche, F., Prieur, F., Enjolras, F., \& Barthelemy, J.C. (1999). Age-associated alteration of muscle oxygenation measured by near infrared spectroscopy during exercise. Archives of Physiology and Biochemistry, 107(2), 159-167.

DeLorey, D.S., Paterson, D.H., \& Kowalchuk, J.M. (2007). Effects of ageing on muscle O2 utilization and muscle oxygenation during the transition to moderate-intensity exercise. Applied Physiology, Nutrition, and Metabolism, $32(6), 1251-1262$.

Ferri, A., Adamo, S., Longaretti, M., Marzorati, M., Lanfranconi, F., Marchi, A., \& Grassi, B. (2007). Insights into central and peripheral factors affecting the "oxidative performance" of skeletal muscle in aging. European Journal of Applied Physiology, 100(5), 571-579.

Gonzalez-Alonso, J., \& Calbet, J.A. (2003). Reductions in systemic and skeletal muscle blood flow and oxygen delivery limit maximal aerobic capacity in humans. Circulation, 107(6), 824-830.

Gordon III, J.A., Hoffman, J.R., Arroyo, E., Varanoske, A.N., Coker, N.A., Gepner, Y., Wells, A.J., Stout, J.R., \& Fukuda, D.H. (2017). Comparisons in the recovery response from resistance exercise between young and middle-aged men. Journal of Strength and Conditioning Research, 31, 3454-3462.

Grassi, B., \& Quaresima, V. (2016). Near-infrared spectroscopy and skeletal muscle oxidative function in vivo in health and disease: A review from an exercise physiology perspective. Journal of Biomedical Optics, 21(9), 091313. Available at: https://www.spiedigitallibrary.org/journals/Journal-of-Biomedical-Optics/volume-21/ issue-9/091313/Near-infrared-spectroscopy-and-skeletal-muscle-oxidative-function-iin-vivo/10.1117/1. JBO.21.9.091313.full?SSO=1 
Groen, B.B., Hamer, H.M., Snijders,T., van Kranenburg, J., Frijns, D., Vink, H., \& van Loon, L.J. (2014). Skeletal muscle capillary density and microvascular function are compromised with aging and type 2 diabetes. Journal of Applied Physiology (1985), 116(8), 998-1005.

Hamaoka, T., McCully, K.K., Quaresima, V., Yamamoto, K., \& Chance, B. (2007). Near-infrared spectroscopy/ imaging for monitoring muscle oxygenation and oxidative metabolism in healthy and diseased humans. Journal of Biomedical Optics, 12(6), 062105. Available at: https://www.spiedigitallibrary.org/journals/Journal-ofBiomedical-Optics/volume-12/issue-6/062105/Near-infrared-spectroscopy-imaging-for-monitoring-muscleoxygenation-and-oxidative/10.1117/1.2805437.short

Hart, C.R., Layec, G., Trinity, J.D., Liu, X., Kim, S.E., Groot, H.J., Le Fur, Y., Sorensen, J.R., Jeong, E.K., \& Richardson, R.S. (2015). Evidence of preserved oxidative capacity and oxygen delivery in the plantar flexor muscles with age. Journals of Gerontology, 70(9), 1067-1076.

Haskell, W.L., Lee, I.M., Pate, R.R., Powell, K.E., Blair, S.N., Franklin, B.A., \& American Heart Association. (2007). Physical activity and public health: Updated recommendation for adults from the American College of Sports Medicine and the American Heart Association. Circulation, 116(9), 1081-1093.

Heinonen, I., Koga, S., Kalliokoski, K.K., Musch, T.I., \& Poole, D.C. (2015). Heterogeneity of muscle blood flow and metabolism: Influence of exercise, aging, and disease states. Exercise and Sport Sciences Reviews, 43(3), 117-124.

Hoffman, J.R., Im, J., Kang, J., Maresh, C.M., Kraemer, W.J., French, D., \& Chance, B. (2007). Comparison of lowand high-intensity resistance exercise on lipid peroxidation: Role of muscle oxygenation. Journal of Strength and Conditioning Research, 21(1), 118-122.

Hoffman, J.R., Im, J., Rundell, K.W., Kang, J., Nioka, S., Spiering, B.A., \& Chance, B. (2003). Effect of muscle oxygenation during resistance exercise on anabolic hormone response. Medicine and Science in Sports and Exercise, 35(11), 1929-1934.

Ichimura, S., Murase, N., Osada, T., Kime, R., Homma, T., Ueda, C., \& Katsumura, T. (2006). Age and activity status affect muscle reoxygenation time after maximal cycling exercise. Medicine and Science in Sports and Exercise, 38(7), 1277-1281.

Jones, B., Hesford, C.M., \& Cooper, C.E. (2013). The use of portable NIRS to measure muscle oxygenation and haemodynamics during a repeated sprint running test. Advances in Experimental Medicine and Biology, 789, 185-191.

Kirkendall, D.T., \& Garrett, W.E.Jr. (1998). The effects of aging and training on skeletal muscle. American Journal of Sports Medicine, 26(4), 598-602.

Koga, S., Rossiter, H.B., Heinonen, I., Musch, T.I., \& Poole, D.C. (2014). Dynamic heterogeneity of exercising muscle blood flow and O2 utilization. Medicine and Science in Sports and Exercise, 46(5), 860-876.

Kutsuzawa, T., Shioya, S., Kurita, D., Haida, M., \& Yamabayashi, H. (2001). Effects of age on muscle energy metabolism and oxygenation in the forearm muscles. Medicine and Science in Sports and Exercise, 33(6), 901-906.

Lin, T.Y., Lin, L.L., Ho, T.C., \& Chen, J.J. (2014). Investigating the adaptation of muscle oxygenation to resistance training for elders and young men using near-infrared spectroscopy. European Journal of Applied Physiology, 114(1), 187-196.

Mancini, D.M., Bolinger, L., Li, H., Kendrick, K., Chance, B., \& Wilson, J.R. (1994). Validation of near-infrared spectroscopy in humans. Journal of Applied Physiology (1985), 77(6), 2740-2747.

Miljkovic, N., Lim, J.Y., Miljkovic, I., \& Frontera, W.R. (2015). Aging of skeletal muscle fibers. Annals of Physical and Rehabilitation Medicine, 39(2), 155-162.

Mitchell, W.K., Williams, J., Atherton, P., Larvin, M., Lund, J., \& Narici, M. (2012). Sarcopenia, dynapenia, and the impact of advancing age on human skeletal muscle size and strength: A quantitative review. Frontiers in Physiology, 3, 260.

Murias, J.M., Kowalchuk, J.M., \& Paterson, D.H. (2010). Speeding of VO2 kinetics with endurance training in old and young men is associated with improved matching of local $\mathrm{O} 2$ delivery to muscle $\mathrm{O} 2$ utilization. Journal of Applied Physiology (1985), 108(4), 913-922.

Pereira, M.I., Gomes, P.S., \& Bhambhani, Y.N. (2007). A brief review of the use of near infrared spectroscopy with particular interest in resistance exercise. Sports Medicine, 37(7), 615-624.

Proctor, D.N., Sinning, W.E., Walro, J.M., Sieck, G.C., \& Lemon, P.W. (1995). Oxidative capacity of human muscle fiber types: Effects of age and training status. Journal of Applied Physiology (1985), 78(6), 2033-2038.

Spencer, M.D., Amano, T., Kondo, N., Kowalchuk, J.M., \& Koga, S. (2014). Muscle O2 extraction reserve during intense cycling is site-specific. Journal of Applied Physiology (1985), 117(10), 1199-1206.

Tsukagoshi, R., Tateuchi, H., Fukumoto, Y., Okumura, H., \& Ichihashi, N. (2012). Stepping exercises improve muscle strength in the early postoperative phase after total hip arthroplasty: A retrospective study. American Journal of Physical Medicine and Rehabilitation, 91(1), 43-52.

van Beest, P.A., van der Schors, A., Liefers, H., Coenen, L.G., Braam, R.L., Habib, N., \& Spronk, P.E. (2012). Femoral venous oxygen saturation is no surrogate for central venous oxygen saturation. Critical Care Medicine, 40(12), 3196-3201. 
Vercruyssen, F., Easthope, C., Bernard, T., Hausswirth, C., Bieuzen, F., Gruet, M., \& Brisswalter, J. (2014). The influence of wearing compression stockings on performance indicators and physiological responses following a prolonged trail running exercise. European Journal of Sport Science, 14(2), 144-150.

Weir, J.P. (2005). Quantifying test-retest reliability using the intraclass correlation coefficient and the SEM. Journal of Strength and Conditioning Research, 19(1), 231-240.

Wells, A.J., Fukuda, D.H., Hoffman, J.R., Gonzalez, A.M., Jajtner, A.R., Townsend, J.R., \& Stout, J.R. (2014). Vastus lateralis exhibits non-homogenous adaptation to resistance training. Muscle and Nerve, 50(5), 785-793.

Submitted: November 3, 2017

Accepted: February 26, 2018

Published Online First: October 19, 2018

Correspondence to:

Yftach Gepner, Ph.D.

School of Public Health, Sackler Faculty of Medicine and Sylvan Adams Sports Institute

Tel Aviv University

Tel Aviv, Israel

Tel.: +972 36409040

E-mail address: gepner@tauex.tau.ac.il 